\title{
COMPARISON OF THE COMPLIOR ANALYSE DEVICE WITH SPHYGMOCOR AND COMPLIOR SP FOR PULSE WAVE VELOCITY AND CENTRAL PRESSURE ASSESSMENT
}

\section{Short title: Validation of the Complior Analyse device}

Francesco STEA ${ }^{1}$, Erwan BOZEC ${ }^{2}$, Sandrine MILLASSEAU ${ }^{3}$, Hakim KHETTAB ${ }^{2}$, Pierre BOUTOUYRIE ${ }^{2}$, Stéphane LAURENT ${ }^{2}$

1 - Internal medicine department, Pisa University, Italie

2 - Inserm U970, Université Paris Descartes, Assistance Publique hôpitaux de Paris, Paris, France

3 - Pulse Wave Consulting,Saint Leu la Foret, France

Correspondence to:

Sandrine Millasseau

Pulse Wave Consulting

$72 \mathrm{~b}$ rue de Montlignon

95320 Saint Leu la Foret

France

Email: sandrine_millasseau@yahoo.fr

Conflicts of interest: Sandrine Millasseau works as a freelance specialist on pulse wave analysis and receives revenues from several medical devices companies including Alam Medical and AtCor Medical whose devices have been used in this work.

Word counts : 4054 (including references - excluding abstracts, title page and figures)

Number of table: 1

Number of figure: 6 


\section{$\underline{\text { Abstract }}$}

The Complior device (Alam Medical, France) was used in epidemiological studies which established pulse wave velocity (PWV) as cardiovascular risk marker. Central pressure is related but complementary to PWV and also associated to cardiovascular outcomes. The new Complior Analyse measures both PWV and central BP during the same acquisition.

The aim of this study was to compare PWV values from Complior Analyse with the previous Complior SP and with Sphygmocor (AtCor, Australia), and to compare central systolic pressure from Complior Analyse and Sphygmocor.

Peripheral and central pressure and PWV were measured with the 3 devices in 112 subjects. PWV measurements from Complior Analyse were analyzed using two footdetection algorithms (PWVca_it and PWVca_cs). Both radial (ao-SBPscr) and carotid (car-SBPscr) approaches from Sphygmocor were compared to carotid Complior Analyse measurements (car-SBPca). Same distance and same calibrating pressures were used for all devices.

PWVca_it was strongly correlated to PWVscr $\left(R^{2}=0.93, p<0.001\right)$ with a difference of $0.0 \pm 0.7 \mathrm{~m} / \mathrm{s}$. PWVca_cs was also correlated to PWVcs $\left(R^{2}=0.90, p<0.001\right)$ with a difference of $0.1 \pm 0.7 \mathrm{~m} / \mathrm{s}$. Central systolic pressures were strongly correlated. The difference between car-SBPca and ao-SBPscr was $3.1 \pm 4.2 \mathrm{mmHg}(\mathrm{p}<0.001)$, statistically equivalent to the difference between car-SBPscr and ao-SBPscr $(3.9 \pm 5.8 \mathrm{mmHg}, p<0.001)$ while the difference between car-SBPca and car-SBPscr was negligible $(-0.7 \pm 5.6 \mathrm{mmHg}, \mathrm{p}=\mathrm{ns})$.

Conclusion: the new Complior Analyse device provides equivalent results for PWV and central pressure values than Sphygmocor and Complior SP. It reaches AAMI 
standard for central BP and grades excellent for PWV on the Artery society criteria. It can be interchanged with existing devices.

Abstract: 250 words 


\section{Short abstract}

The new Complior Analyse device allows simultaneous measurement of pulse wave velocity (PWV) and central pressure (cBP) during a single acquisition. This study compared PWV values obtained with the new Complior Analyse with the previous Complior SP device and to the Sphygmocor device for pulse wave velocity (PWV) values in 112 subjects with a wide range of PWV. It reaches "excellent" grade on the "Artery" society criteria for both comparison. In the same cohort, Complior Analyse cBP was compared to non-invasive cBP estimated with Sphygmocor. Complior Analyse reaches AAMI standard for systolic cBP.

Short abstract: 93 words

Keywords: arterial stiffness; pulse wave velocity; central pressure; Complior; validation 


\section{Introduction:}

Aortic stiffness measured by carotid-femoral pulse wave velocity (PWV) has proven its clinical importance as an independent marker of cardiovascular risk and mortality in the general population[1-7] and in a variety of disease conditions including renal failure[8-14], diabetes [15], stroke[16-18] and hypertension[19-21]. Most of these epidemiological studies have be performed with the Complior device (Alam Medical, France) $[1,4,5,9,12,14,18-20]$ as it provides rapid and reproducible assessment of PWV[22, 23]. Complior uses simultaneous carotid and femoral measurements as advised by the Artery Society [24]. Although widely used for PWV measurement, sensors used in the previous version were not enough responsive for performing pressure wave analysis. In this latest evolution, Complior Analyse (ca) is equipped with high quality pressure sensors which have been shown to record invasive signals accurately[25]. During standard PWV measurement, it is hence now possible to assess central pressure directly from carotid waveforms. Carotid pressure waveforms have been shown be quite similar to aortic pressure waves[26, 27] and 3 out of 6 studies showing that central pressure better predicts cardiovascular mortality than peripheral pressure have been performed from carotidograms[28-30], 2 from invasive aortic waveforms[31, 32] and only 1 from estimated aortic waveforms[33].

The previous version of Complior has been validated regarding PWV values[22, 34, 35]. Since then, the Artery society has produced guidelines and a protocol to validate non-invasive PWV devices[24]. However to date no standardised protocol exists to validate measurement of central pressure. As sensors and the algorithm to detect the foot of the waveform have been changed, the first aim of the present study was to estimate the ability of the Complior Analyse device to measure PWV following the protocol from the Artery society. The second objective was to compare central 
systolic pressure values measured with Complior Analyse with non-invasive assessments of central pressure with the Sphygmocor CPV device (AtCor, Australia).

\section{Methods:}

112 subjects were recruited subsequently from the Artery Research Laboratory in European Hospital of George Pompidou. Measurements were performed part of clinical research studies or part of a routine arterial screening procedure including among other thing, central pressure, carotid-femoral PWV, carotid intima-media thickness and carotid wall characterization. All patients gave written informed consent.

\section{$\underline{P W V \text { study }}$}

After 10 minutes of supine rest, peripheral blood pressure was measured every $3 \mathrm{~min}$ to check haemodynamic stability. Carotid femoral PWV was then measured alternatively by trained operators (FS et EB) in triplicate with the previous Complior SP device (PWVcs), with the Sphygmocor (PWVscr) and with the new Complior Analyse device (PWVca). Single recordings were deemed acceptable is the SD of the PWV recording was below $0.5 \mathrm{~m} / \mathrm{s}$. Distance was assessed as 0.8 times the direct surface measurement between the carotid and femoral arterial sites as now recommended[36]. The same distance was used for the 3 devices.

Both Complior SP and Sphygmocor were used as comparison devices. Indeed Complior SP was used in numerous epidemiological studies $[1,4,5,9,12,14,18$ 20]. It measured simultaneous distension waveforms sampled at $4 \mathrm{kHz}$ on $12 \mathrm{bits}$ and qualified as the recommended non-invasive reference for the Artery Society protocol[24]. However the foot of the waveforms is detected with a validated 
proprietary algorithm based on the second derivative and auto-correlation[22]. This algorithm has been found to differ from the now recommend intersecting tangent algorithm especially for higher value of PWV[37].

The Sphygmocor system (v8.2, AtCor, Australia) uses ECG-gated tonometric measurement sampled at $128 \mathrm{~Hz}$ on 12bits and the intersecting tangent algorithm. As such it only qualified as "secondary reference" in the Artery Society guidelines[24]. The new Complior Analyse system uses simultaneous pressure signals sampled at 1 $\mathrm{kHz}$ on 16 bits and allows both algorithms to be used. Complior Analyse PWV values with SP algorithm (PWVca_cs) was hence compared to Complior SP PWV values (PWVcs) while values from the Complior Analyse with the intersecting tangent algorithm (PWVca) were compared to Sphygmocor PWV values (PWVscr).

\section{Central pressure study}

Radial and carotid tonometry with Sphygmocor were performed to assess central pressure. Radial tonometry waveforms were calibrated to brachial cuff systolic and diastolic pressures performed immediately before and used to estimate aortic systolic pressure with the Sphygmocor's generalized transfer function (ao-SBPscr). Carotid waveforms were calibrated to cuff diastolic pressure and mean pressure assessed from the area under the radial curve[38]. No transfer function was applied on the carotid waveforms and carotid systolic pressure was obtained from direct calibration of the carotid waveform (car-SBPscr) using mean and diastolic blood pressure. Carotid systolic pressure from Complior Analyse (car-SBPca) was obtained from carotid traces acquired during the PWV assessment. It has to be highlighted that aoSBPscr is an estimation of the aortic arch systolic pressure, while car-SBPscr and 
car-SBPca are direct measures at the carotid level. While carotid and aortic pressure are usually both referred as central pressure, it is important to note that they are actually from different anatomical sites. In order to compare accuracy of signal recordings, Complior Analyse and Carotid Sphygmocor traces were calibrated using the same brachial cuff diastolic and integrated mean radial pressures. All measures were done in triplicate.

\section{Statistics:}

For each subjects the average of the 3 recordings taken with each device was calculated. The coefficient of variation was calculated as averaged subject mean standard deviation (SD) divided by the subject mean value and expressed as a percentage.

Scatter plot and Pearson's correlation coefficient was used to check correlation between variables. Bland Altman plot were used to assess the amplitude of the difference and check the absence of trend across the value range. The accuracy criteria for PWV was taken from the Artery Society guidelines document[24]: PWV measurement were classified as "excellent" if the mean difference is below $0.5 \mathrm{~m} / \mathrm{s}$ and the $\mathrm{SD}$ of the difference $0.8 \mathrm{~m} / \mathrm{s}$ and as "acceptable" if the mean difference $<1.0 \mathrm{~m} / \mathrm{s}$ and $\mathrm{SD} \leq 1.5 \mathrm{~m} / \mathrm{s}$.

The standard requirements for the non-invasive brachial BP monitors from the Association for the Advancement of Medical Instrumentation (AAMI)[39] was used to evaluate the goodness of agreement between central systolic pressure values: mean difference $<5 \mathrm{mmHg}$ and $\mathrm{SD}<8 \mathrm{mmHg}$. 


\section{Results:}

\section{$\underline{P W V \text { validation }}$}

3 subjects were discarded for PWV analysis for failure to obtain in triplicate with one of the 3 devices good quality carotid recordings which prevent correct foot detection (1 per device). 2 were further discarded because blood pressure and/or heart rate varied by more than $10 \mathrm{mmHg}$ or bpm between devices.

Characteristics of the remaining 107 subjects are shown on table 1 .

Coefficient of variation for PWVscr values was $5.25 \%, 4 \%$ for PWVcs, $3.4 \%$ for PWVca with the intersecting tangent algorithm and $3.0 \%$ for PWVca with the SP algorithm.

As expected from the literature[37, 40], PWVscr and PWVcs were strongly correlated $\left(R^{2}=0.87, p<0.001\right)$ but they were different especially for higher value of PWV with Complior SP underestimating PWV compared to Sphygmocor as shown on figure 1 (overall mean difference $=-0.3 \pm 0.9 \mathrm{~m} / \mathrm{s}, \mathrm{p}=\mathrm{ns}$ ). The accuracy between this 2 devices reached "acceptable" on the Artery society guidelines[24].

PWVca with the intersecting tangent algorithm was strongly correlated with PWVscr $\left(R^{2}=0.93, p<0.001\right)$ with very little difference (error $\left.=0.0 \pm 0.7 \mathrm{~m} / \mathrm{s}, \mathrm{p}=\mathrm{ns}\right)$ and no trend across the PWV range (see figure 2). This rated "excellent" on the accuracy criteria from the Artery society[24].

PWVca with the SP algorithm was also strongly correlated with PWVcs $\left(R^{2}=0.90\right.$, $p<0.001)$. The difference and its standard deviation between the previous and the latest version of Complior were $0.1 \pm 0.8 \mathrm{~m} / \mathrm{s}(p=n s)$ reaching the "excellent" grade (figure 3). 


\section{Central pressure comparison}

14 subjects were discarded due to inability to get good quality carotid recordings in triplicate with stable peripheral blood pressure: 9 with the Sphygmocor device, 6 with the Complior Analyse device, including 2 on both devices.

Characteristics of the remaining 98 subjects are shown on table 1. Measurements of central systolic pressures from direct carotid traces with Sphygmocor (car-SBPscr) and Complior Analyse (car-SBPca) showed very strong correlation $\left(R^{2}=0.94, p<0.001\right.$, figure 4). The difference and its standard deviation was small and fulfilled the AAMI criteria $(-0.7 \pm 5.6 \mathrm{mmHg}, p=n s)$.

When compared to aortic pressure estimated from radial waveforms with Sphygmocor (ao-SBPscr), car-SBPca was also strongly correlated $\left(R^{2}=0.96\right.$, $p<0.001$, figure 5) but the Bland-Altman analysis showed a small significant difference between the two anatomically different sites (error $=3.1 \pm 4.2 \mathrm{mmHg}$, $p<0.001$, figure 5). This difference was of the same magnitude than the one observed between carotid and estimated aortic from Sphygmocor $\left(R^{2}=0.96, p<0.001\right.$, error $=$ $3.7 \pm 5.5, p<0.001$, figure 6 ). However they both still fulfill the AAMI criteria.

\section{Discussion:}

This study showed that the new Complior Analyse device provides similar PWV results than the Sphygmocor CPV device or than the previous Complior SP device when a matching algorithm is used. Central pressure measurement from the carotid with Complior Analyse was also comparable to non-invasive Sphygmocor estimates. 
The Complior SP device was used in most epidemiological studies $[1,4,5,9,12,14$, 18-20]. It has been validated in the past when the recommended foot detection algorithm was not the intersecting tangent[22, 34, 35]. Our results confirm that the new Complior Analyse system is substitutable to older versions of Complior SP when using the same algorithm.

PWV can be assessed with simultaneous measurement as with Complior or alternatively, successive ECG gated acquisitions can be performed. These 2 approaches have been found equivalent when hemodynamic state is stable[41]. However our results showed that the coefficient of variation for repeated measures was slightly higher with ECG gated measurements (CV = 5.25\% for PWVscr) than with simultaneous measurements ( $\mathrm{CV}=3.4 \%$ for PWVca with intersecting tangent algorithm) even in highly controlled conditions where blood pressure and heart rate were very stable.

Several algorithms have been proposed to detect the foot of the waveform[22, 42, 43]. They are unfortunately not equivalent especially regarding higher values of PWV $[37,43,44]$. However, as it is less sensitive to measurements artefact and shows the least variability[42], the Artery Society[24] and the Expert consensus on arterial stiffness[36] recommend to use the "intersecting tangent" algorithm. This algorithm is now implemented in the latest version of the Complior system and shows quasi perfect agreement with the Sphygmocor v8.2 device with no trend across PWV range (right panel, figure 2). A recent publication [45] comparing carotid to leg cuff approach to Sphygmocor CPV showed an overall small difference with however much higher discrepancy for higher value and of PWV questioning its applicability in higher risk patients. 
After foot-detection algorithm, the second parameter influencing greatly PWV is the arterial distance. Because our aim was to compare techniques, we chose to use the same distance for each device. Indeed, when comparing two techniques, the use of the correct distance is less important than using identical distance values for the 3 devices. Using a different distance methodology (caliper instead of tape meter for example) would only have modified absolute values of PWV. Similar results are obtained by comparing directly transit time. It would not change our conclusion that Complior Analyse PWV measure reaches "excellent" on the Artery Society criteria. As recommended, our study population had a large range of age and blood pressure, however only $25 \%$ of the study group have an elevated PWV value ( $>10 \mathrm{~m} / \mathrm{s})$. Hence our results might not be generalised to a population of patients with very stiff arteries. With its new pressure sensors, Complior Analyse now records carotid pressure waveforms during the standard PWV measurement.

The accuracy to correctly monitor invasive pressure traces has been published elsewhere with good agreement between harmonics content of invasive and Complior Analyse waveform[25]. Sphygmocor tonometer also showed similar frequency content than invasive waveforms[46]. The quality of central pressure assessment relies on the fidelity of recording of the pressure wave, coupled with adequate calibration. Because of increased capacity of $A D$ converters, compared to SphygmoCor, Complior Analyse allows a 8x increase in time resolution and improved dynamics by $10 \mathrm{~dB}$, which might contribute to the good performance of this device. Both Complior Analyse and SphygmoCor devices use applanation tonometry which provides uncalibrated waveforms whose amplitude and offset depends on the holdon pressure, on the underlying tissue characteristics and on intra-arterial pressure. 
Our results here show that provided similar calibration, Complior Analyse gives similar central systolic pressure than Sphygmocor. The Sphygmocor device has been extensively validated and shows good agreement with invasive pressure when invasively calibrated[47].

We have shown that there is an excellent agreement between SphygmoCor and Complior Analysis devices in term of waveform analysis and we obtained values of central pressure that are practically similar. Thus both device are interchangeable. Our results also highlight the presence of a small difference between aortic and carotid systolic pressure which is expected on physiological grounds. While carotid and ascending arteries are geographically and functionally very close, the $10-15 \mathrm{~cm}$ difference between the 2 sites might explain amplification by $3 \mathrm{mmHg}$ between carotid and aortic pressures. Invasive studies comparing carotid and aortic waveforms did not focus on central systolic pressure[26, 27], however Salvi et al.[41] reported a difference of $2.5 \mathrm{mmHg}$ between the 2 sites which is comparable to our results. In their paper comparing invasive pressures with carotid distension waveforms, Van Bortel et al. also found similar difference $(3.8 \mathrm{mmHg})[38]$. This difference is small and falls well within the AAMI criteria. It does not invalidate mortality studies showing that central pressure is a stronger predictor of cardiovascular events and mortality than peripheral pressure[28-30]. Despite that the carotid approach is the one the most used in mortality studies, it has to been mentioned that good quality carotid waveforms can be difficult to obtain with tonometry. Indeed, the absence of underlying bony structure, the presence of the soft tissues and the proximity of the trachea can make the measurement difficult as shown by the fact we had to discard $10 \%$ (14 out of our 112) of the subjects due to inability to perform measurements triplicate with both device. Here we were able to use the 'real' mean blood pressure 
by integrating radial blood pressure. Because there are small but quantifiable differences between brachial and radial pressure waveforms form factors, and because applanation of radial artery might not be routinely performed with Complior Analyse, the use of calculated mean arterial pressure might induce larger discrepancies with alternative methods.

The new Complior Analyse system is equivalent to Complior SP or Sphygmocor devices when the appropriate algorithm is used and it grades "excellent" on the Artery society criteria. Carotid-femoral PWV is a recommended parameter to assess organ damage and cardiovascular risk in the management of arterial hypertension[48]. Our results show that the medical device industry is actively working to provide accurate and reliable systems to the market. This will surely contribute to the expansion of the measurement of arterial stiffness in routine patient risk assessment.

Complior Analyse new pressure sensor with appropriate calibration allows to measure accurately central systolic pressure directly from the carotid with no assumption or mathematical modelling. It could hence be used in research studies and clinical routine especially in subjects when the application of a transfer function is questionable such as children or patients with upper arm arterial calcification. 
Table 1: Patients characteristics

\begin{tabular}{lcc}
\hline & mean \pm sd & range \\
\hline \multicolumn{1}{l}{ PWV validation $n=107$ (55 men-52 women) } & & \\
Age (yrs) & $47.2 \pm 15.7$ & $16-83$ \\
BMI (kg/m $\left.{ }^{2}\right)$ & $24.5 \pm 4.2$ & $17.4-35.4$ \\
SBP (mmHg) & $128 \pm 51$ & $91-224$ \\
DBP (mmHg) & $70 \pm 11$ & $46-108$ \\
HR (bpm) & $66 \pm 10$ & $45-95$ \\
PWV Sphygmocor & $8.6 \pm 2.5$ & $5.1-19.9$ \\
PWV Complior SP & $8.2 \pm 2.1$ & $4.9-17.6$ \\
PWV Complior Analyse intersecting & $8.6 \pm 2.6$ & $5.3-19.4$ \\
tangents algorithm & & \\
PWV Complior analyse peak of second & $8.3 \pm 2.4$ & $5.2-18.3$ \\
derivative algorithm & & \\
Central Pressure validation n=98 (49 men and 49 women) & \\
Age (yrs) & $47.4 \pm 15.4$ & $18-83$ \\
BMI (kg/m ${ }^{2}$ ) & $24.6 \pm 4.2$ & $17.6-35.4$ \\
Peripheral SBP (mmHg) & $131 \pm 20$ & $100-236$ \\
Peripheral DBP (mmHg) & $73 \pm 11$ & $53-116$ \\
Peripheral MBP (mmHg) & $92 \pm 15$ & $67-163$ \\
HR (bpm) & $66 \pm 10$ & $44-94$ \\
Aortic SBP (Sphygmocor, transfer & $117 \pm 21$ & $84-220$ \\
function) & & \\
Carotid SBP (Sphygmocor) & $121 \pm 24$ & $83-246$ \\
Carotid SBP (Complior Analyse) & $120 \pm 22$ & $88-232$ \\
\hline
\end{tabular}


Figure 1:

Comparison of pulse wave velocity values from Sphygmocor device (PWVscr) and the Complior SP device (PWVcs). Left: Scatter plot with regression line (solid line). The dotted line represents the identity line.

Right: Bland-Altman plot of the difference.
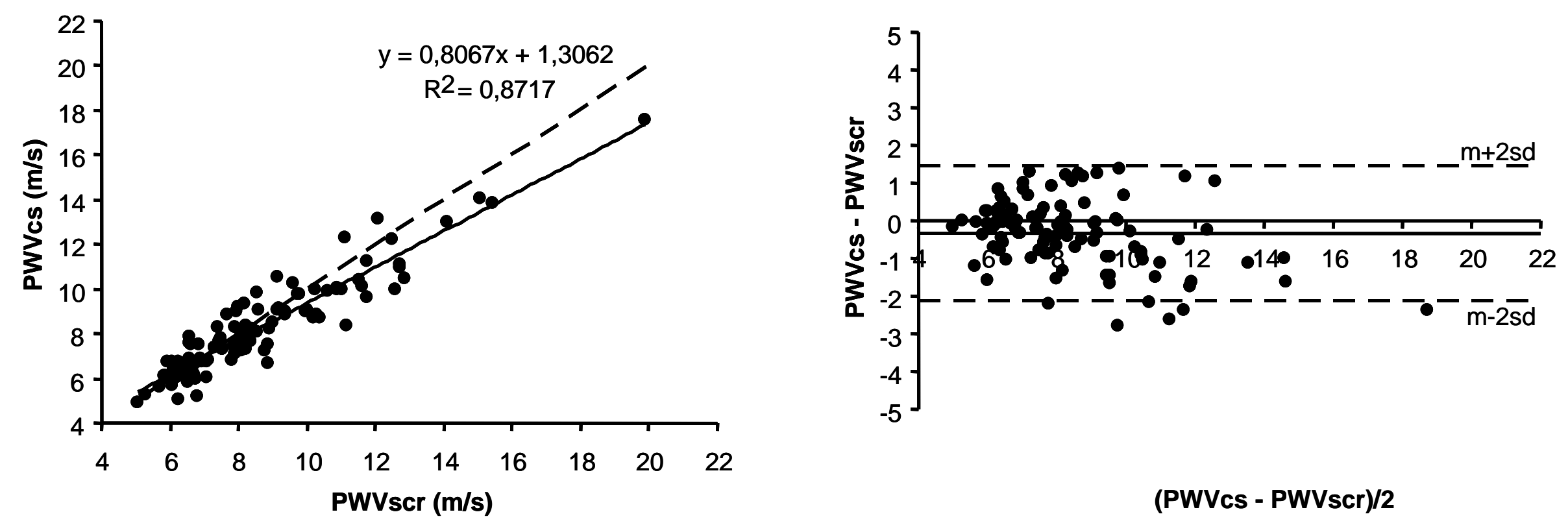

(PWVcs - PWVscr)/2 
Figure 2:

Comparison of pulse wave velocity values from Sphygmocor device (PWVscr) and the new Complior Analyse device with the intersecting tangent algorithm (PWVca_it).

Left: Scatter plot with regression line (solid line). The identity line is superimposed with the regression line and hence not visible.

Right: Bland-Altman plot of the difference.
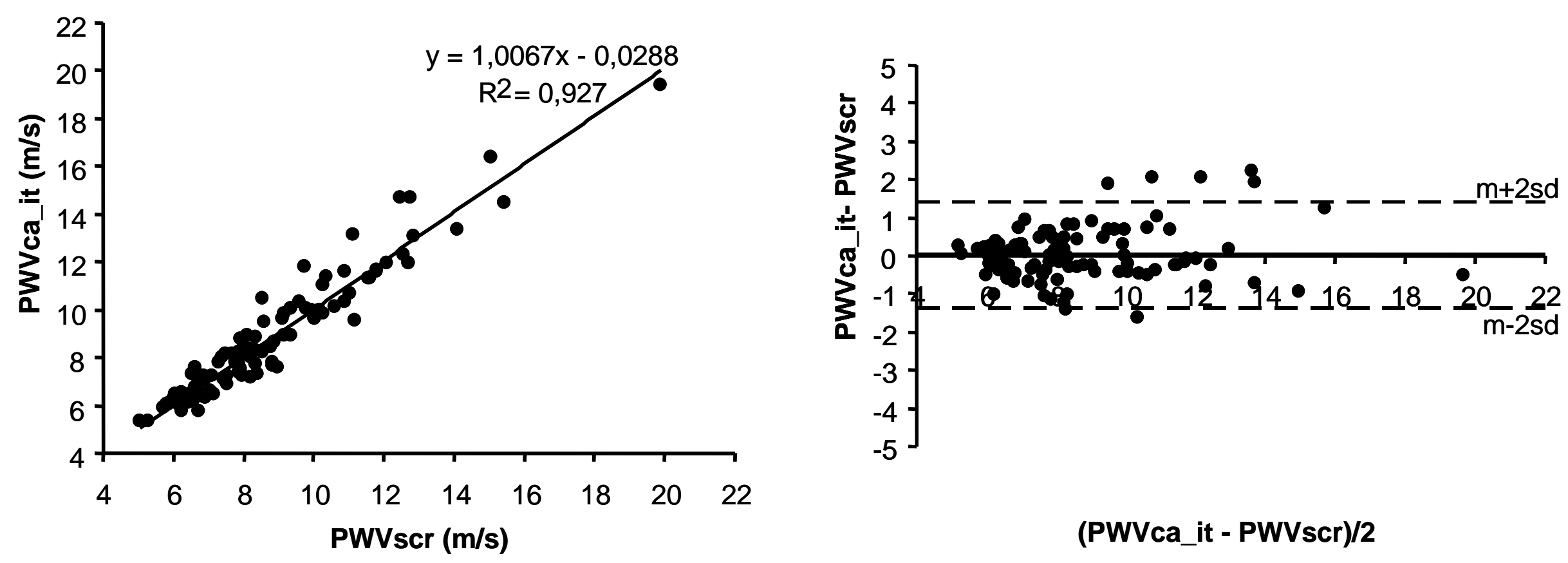

(PWVca_it - PWVscr)/2 
Figure 3:

Comparison of pulse wave velocity values from Complior SP device (PWVcs) and the new Complior Analyse device with the SP algorithm (PWVca_cs).

Left: Scatter plot with regression line (solid line). The dotted line represents the identity line.

Right: Bland-Altman plot of the difference.
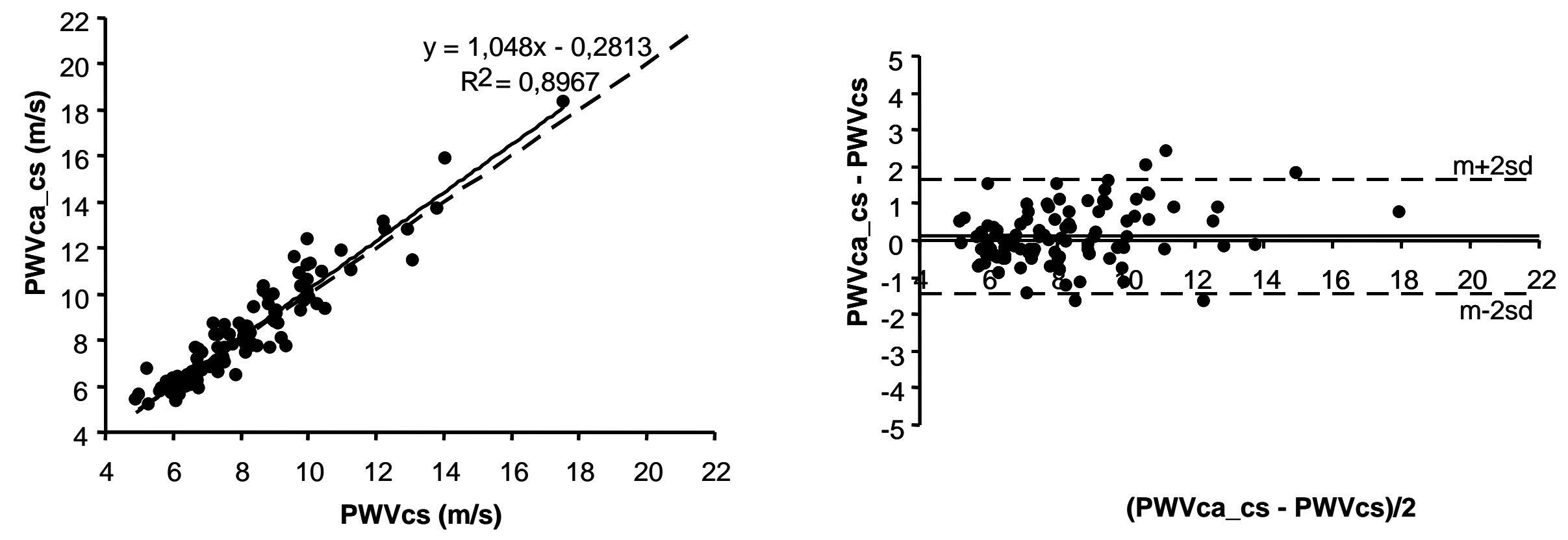

(PWVca_cs - PWVcs)/2 
Figure 4:

Comparison of central systolic pressure measured from Sphygmocor carotid traces (car-SBPscr) and Complior Analyse carotid traces (car-SBPca)

Left: scatter plot with regression line (solid line)

Right: bland-Altman plot of the difference
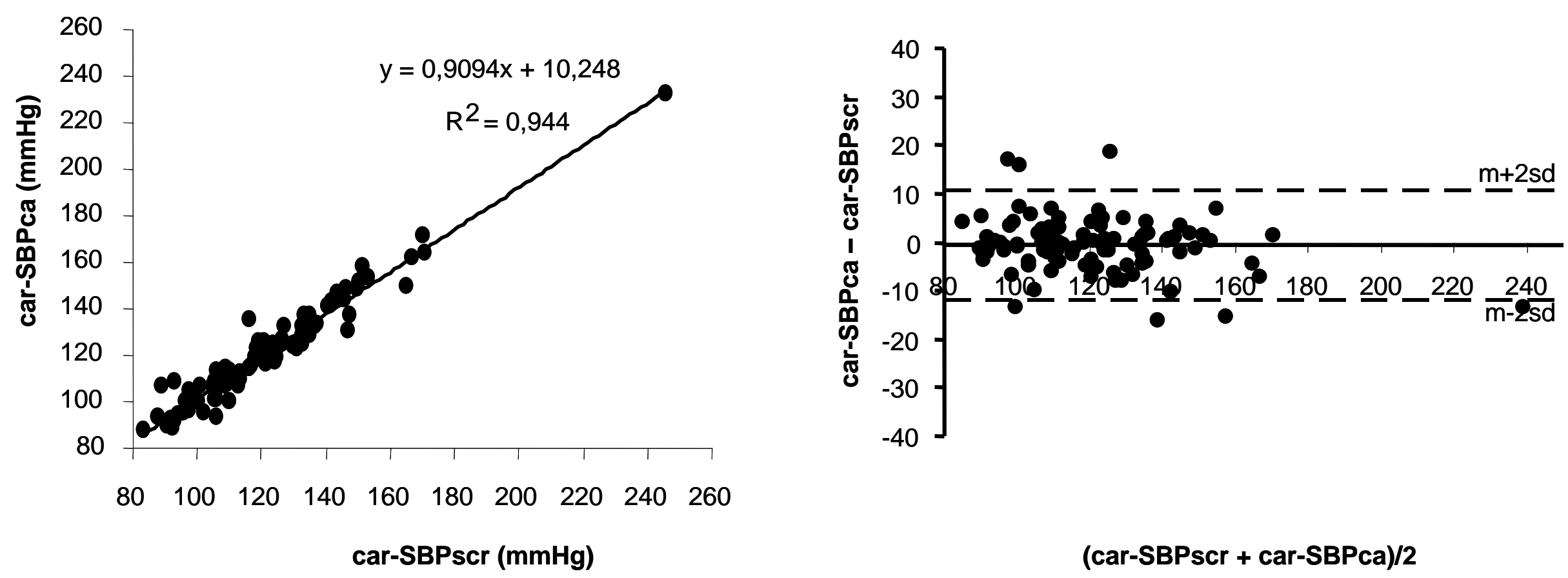

$($ car-SBPscr + car-SBPca)/2 
Figure 5

Comparison of central systolic pressure estimated from Sphygmocor radial traces (ao-SBPscr) and Complior Analyse carotid traces (car-SBPca)

Left: scatter plot with regression line (solid line)

Right: bland-Altman plot of the difference
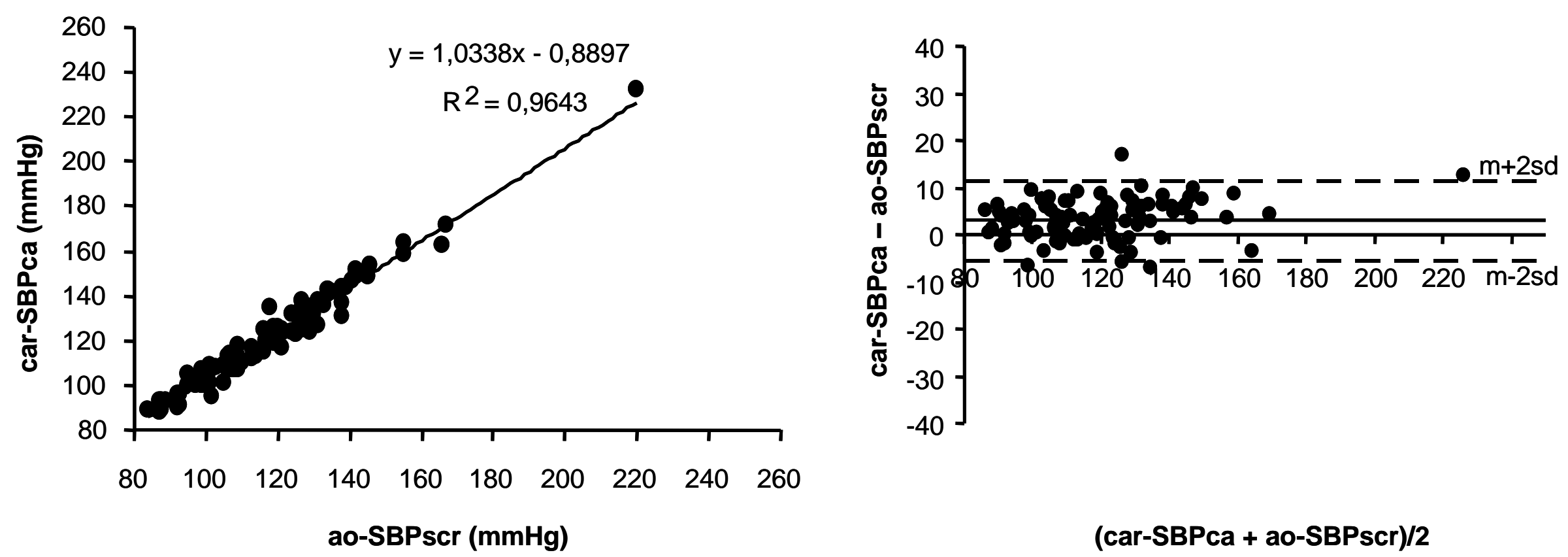

$($ car-SBPca + ao-SBPscr)/2 
Figure 6

Comparison of central systolic pressure measured from Sphygmocor carotid traces (car-SBPscr) and estimated from Sphygmocor radial traces (ao-SBPscr)

Left: scatter plot with regression line (solid line)

Right: bland-Altman plot of the difference
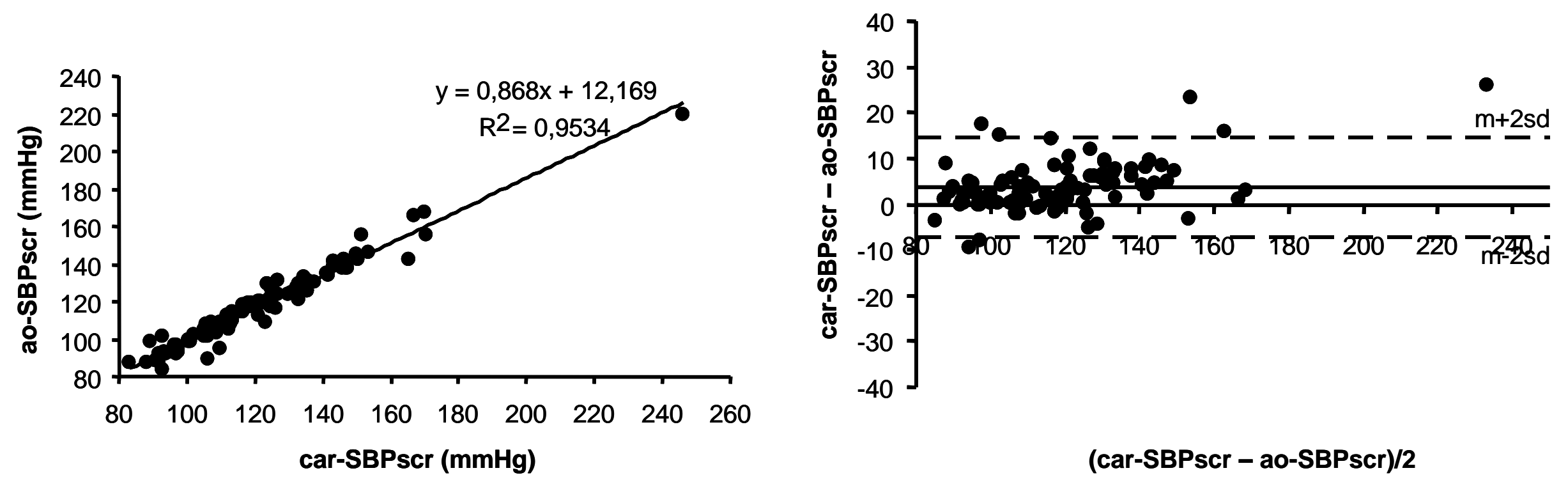

(car-SBPscr - ao-SBPscr)/2 


\section{Reference List}

1. Mattace-Raso FU, van der Cammen TJ, Hofman A, van Popele NM, Bos ML, Schalekamp MA, et al. Arterial stiffness and risk of coronary heart disease and stroke: the Rotterdam Study. Circulation 2006; 113:657-663.

2. Wang KL, Cheng HM, Sung SH, Chuang SY, Li CH, Spurgeon HA, et al. Wave reflection and arterial stiffness in the prediction of 15-year all-cause and cardiovascular mortalities: a community-based study. Hypertension 2010; 55:799-805.

3. Willum-Hansen T, Staessen JA, Torp-Pedersen C, Rasmussen S, Thijs L, Ibsen H, et al. Prognostic value of aortic pulse wave velocity as index of arterial stiffness in the general population. Circulation 2006; 113:664-670.

4. Maldonado J, Pereira T, Polonia J, Silva JA, Morais J, Marques M. Arterial stiffness predicts cardiovascular outcome in a low-to-moderate cardiovascular risk population: the EDIVA (Estudo de DIstensibilidade VAscular) project. $J$ Hypertens 2011; 29:669-675.

5. Meaume S, Benetos A, Henry OF, Rudnichi A, Safar ME. Aortic pulse wave velocity predicts cardiovascular mortality in subjects $>70$ years of age. Arterioscler Thromb Vasc Biol 2001; 21:2046-2050.

6. Sutton-Tyrrell K, Najjar SS, Boudreau RM, Venkitachalam L, Kupelian V, Simonsick EM, et al. Elevated aortic pulse wave velocity, a marker of arterial stiffness, predicts cardiovascular events in well-functioning older adults. Circulation 2005; 111:3384-3390.

7. Anderson SG, Sanders TA, Cruickshank JK. Plasma fatty acid composition as a predictor of arterial stiffness and mortality. Hypertension 2009; 53:839-845.

8. Blacher J, Guerin AP, Pannier B, Marchais SJ, Safar ME, London GM. Impact of aortic stiffness on survival in end-stage renal disease. Circulation 1999; 99:2434-2439.

9. Pannier B, Guerin AP, Marchais SJ, Safar ME, London GM. Stiffness of capacitive and conduit arteries: prognostic significance for end-stage renal disease patients. Hypertension 2005; 45:592-596.

10. Shoji T, Emoto M, Shinohara K, Kakiya R, Tsujimoto Y, Kishimoto H, et al. Diabetes mellitus, aortic stiffness, and cardiovascular mortality in end-stage renal disease. J Am Soc Nephrol 2001; 12:2117-2124.

11. Zoungas S, Cameron JD, Kerr PG, Wolfe R, Muske C, McNeil JJ, et al. Association of carotid intima-medial thickness and indices of arterial stiffness with cardiovascular disease outcomes in CKD. Am J Kidney Dis 2007; 50:622-630.

12. Karras A, Haymann JP, Bozec E, Metzger M, Jacquot C, Maruani G, et al. Large Artery Stiffening and Remodeling Are Independently Associated With All- 
Cause Mortality and Cardiovascular Events in Chronic Kidney Disease. Hypertension 2012.

13. Verbeke F, Van BW, Honkanen E, Wikstrom B, Jensen PB, Krzesinski JM, et al. Prognostic value of aortic stiffness and calcification for cardiovascular events and mortality in dialysis patients: outcome of the calcification outcome in renal disease (CORD) study. Clin J Am Soc Nephrol 2011; 6:153-159.

14. Szeto CC, Kwan BC, Chow KM, Leung CB, Law MC, Li PK. Prognostic value of arterial pulse wave velocity in peritoneal dialysis patients. Am J Nephrol 2012; 35:127-133.

15. Cruickshank K, Riste L, Anderson SG, Wright JS, Dunn G, Gosling RG. Aortic pulsewave velocity and its relationship to mortality in diabetes and glucose intolerance: an integrated index of vascular function? Circulation 2002; 106:2085-2090.

16. Gasecki D, Rojek A, Kwarciany M, Kowalczyk K, Boutouyrie P, Nyka W, et al. Pulse wave velocity is associated with early clinical outcome after ischemic stroke. Atherosclerosis 2012; 225:348-352.

17. Gasecki D, Rojek A, Kwarciany M, Kubach M, Boutouyrie P, Nyka W, et al. Aortic stiffness predicts functional outcome in patients after ischemic stroke. Stroke 2012; 43:543-544.

18. Laurent S, Katsahian S, Fassot C, Tropeano AI, Gautier I, Laloux B, et al. Aortic stiffness is an independent predictor of fatal stroke in essential hypertension. Stroke 2003; 34:1203-1206.

19. Boutouyrie P, Tropeano AI, Asmar R, Gautier I, Benetos A, Lacolley P, et al. Aortic stiffness is an independent predictor of primary coronary events in hypertensive patients: a longitudinal study. Hypertension 2002; 39:10-15.

20. Laurent S, Boutouyrie P, Asmar R, Gautier I, Laloux B, Guize L, et al. Aortic stiffness is an independent predictor of all-cause and cardiovascular mortality in hypertensive patients. Hypertension 2001; 37:1236-1241.

21. Terai M, Ohishi M, Ito N, Takagi T, Tatara Y, Kaibe M, et al. Comparison of arterial functional evaluations as a predictor of cardiovascular events in hypertensive patients: the Non-Invasive Atherosclerotic Evaluation in Hypertension (NOAH) study. Hypertens Res 2008; 31:1135-1145.

22. Asmar R, Benetos A, Topouchian J, Laurent P, Pannier B, Brisac A-M, et al. Assessment of arterial distensibility by automatic pulse wave velocity measurement: validation and clinical application studies. Hypertension 1995; 26:485-490.

23. Asmar R, Topouchian J, Pannier B, Benetos A, Safar M. Pulse wave velocity as endpoint in large-scale intervention trial. The Complior study. Scientific, Quality Control, Coordination and Investigation Committees of the Complior Study. J Hypertens 2001; 19:813-818. 
24. Wilkinson IB, McEniery CM, Schillaci G, Boutouyrie P, Segers P, Donald A, et al. Artery Society guidelines for validation of non-invasive haemodynamic measurement devices: Part 1, arterial pulse wave velocity. Artery Research 2010; 4:34-40.

25. Sztrymf B, Jacobs F, Chemla D, Richard C, Millasseau SC. Validation of the new Complior sensor to record pressure signals non-invasively. J Clin Monit Comput 2013.

26. Chen CH, Ting CT, Nussbacher A, Nevo E, Kass DA, Pak P, et al. Validation of carotid artery tonometry as a means of estimating augmentation index of ascending aortic pressure. Hypertension 1996; 27:168-175.

27. Kelly RP, Karamanoglu M, Gibbs H, Avolio AP, O'Rourke MF. Noninvasive Carotid Pressure Wave registration as an indicator of ascending aortic pressure. Journal of Vascular Medicine and biology 1989; 1:241-247.

28. Safar ME, Blacher J, Pannier B, Guerin AP, Marchais SJ, Guyonvarc'h PM, et al. Central pulse pressure and mortality in end-stage renal disease. Hypertension 2002; 39:735-738.

29. Pini R, Cavallini MC, Palmieri V, Marchionni N, Di BM, Devereux RB, et al. Central but not brachial blood pressure predicts cardiovascular events in an unselected geriatric population: the ICARe Dicomano Study. J Am Coll Cardiol 2008; 51:2432-2439.

30. Wang KL, Cheng HM, Chuang SY, Spurgeon HA, Ting CT, Lakatta EG, et al. Central or peripheral systolic or pulse pressure: which best relates to target organs and future mortality? J Hypertens 2009; 27:461-467.

31. Chirinos JA, Zambrano JP, Chakko S, Veerani A, Schob A, Willens HJ, et al. Aortic pressure augmentation predicts adverse cardiovascular events in patients with established coronary artery disease. Hypertension 2005; 45:980-985.

32. Jankowski P, Kawecka-Jaszcz K, Czarnecka D, Brzozowska-Kiszka M, Styczkiewicz $\mathrm{K}$, Styczkiewicz M, et al. Ascending aortic, but not brachial blood pressurederived indices are related to coronary atherosclerosis. Atherosclerosis 2004; 176:151-155.

33. Roman MJ, Devereux RB, Kizer JR, Lee ET, Galloway JM, Ali T, et al. Central pressure more strongly relates to vascular disease and outcome than does brachial pressure: the Strong Heart Study. Hypertension 2007; 50:197-203.

34. Pereira T, Maldonado J. Comparative study of two generations of the Complior device for aortic pulse wave velocity measurements. Blood Press Monit 2010; 15:316321.

35. Podolec P, Kopec G, Podolec J, Wilkolek P, Krochin M, Rubis P, et al. Aortic pulse wave velocity and carotid-femoral pulse wave velocity: similarities and discrepancies. Hypertens Res 2007; 30:1151-1158. 
36. Van Bortel LM, Laurent S, Boutouyrie P, Chowienczyk P, Cruickshank JK, De BT, et al. Expert consensus document on the measurement of aortic stiffness in daily practice using carotid-femoral pulse wave velocity. J Hypertens 2012; 30:445448.

37. Millasseau SC, Stewart AD, Patel SJ, Redwood SR, Chowienczyk PJ. Evaluation of carotid-femoral pulse wave velocity: influence of timing algorithm and heart rate. Hypertension 2005; 45:222-226.

38. Van Bortel LM, Balkestein EJ, van der Heijden-Spek JJ, Vanmolkot FH, Staessen JA, Kragten JA, et al. Non-invasive assessment of local arterial pulse pressure: comparison of applanation tonometry and echo-tracking. J Hypertens 2001; 19:1037-1044.

39. Association for the Advancement of Medical Instrumentation. American national standard: electronic or automated sphygmomanometers. AAMI Arlingon VA 2009.

40. Arterial Stiffness Collaboration group. Determinants of pulse wave velocity in healthy people and in the presence of cardiovascular risk factors: 'establishing normal and reference values'. Eur Heart J 2010.

41. Salvi P, Lio G, Labat C, Ricci E, Pannier B, Benetos A. Validation of a new noninvasive portable tonometer for determining arterial pressure wave and pulse wave velocity: the PulsePen device. J Hypertens 2004; 22:2285-2293.

42. Chiu YC, Arand PW, Shroff SG, Feldman T, Carroll JD. Determination of pulse wave velocities with computerized algorithms. Am Heart J 1991; 121:1460-1470.

43. Vardoulis O, Papaioannou TG, Stergiopulos N. Validation of a novel and existing algorithms for the estimation of pulse transit time: Advancing the accuracy in pulse wave velocity measurement. Am J Physiol Heart Circ Physiol 2013.

44. Salvi P, Magnani E, Valbusa F, Agnoletti D, Alecu C, Joly L, et al. Comparative study of methodologies for pulse wave velocity estimation. J Hum Hypertens 2008.

45. Butlin M, Qasem A, Battista F, Bozec E, McEniery CM, Millet-Amaury E, et al. Carotid-femoral pulse wave velocity assessment using novel cuff-based techniques: comparison with tonometric measurement. J Hypertens 2013.

46. Kelly RP, Hayward C, Ganis J, Daley J, Avolio A, O'Rourke MF. Noninvasive registration of the arterial pressure pulse waveform; using high-fidelity applanation tonometry. Journal of Vascular Medicine and biology 1989; $1: 142-149$.

47. Cheng HM, Lang D, Tufanaru C, Pearson A. Measurement accuracy of non-invasively obtained central blood pressure by applanation tonometry: A systematic review and meta-analysis. Int J Cardiol 2012.

48. Mancia G, Fagard R, Narkiewicz K, Redon J, Zanchetti A, Bohm M, et al. 2013 ESH/ESC Guidelines for the management of arterial hypertension: The Task Force for the management of arterial hypertension of the European Society of 
Hypertension (ESH) and of the European Society of Cardiology (ESC). Eur Heart J 2013. 